\title{
Delegation in Role-Based Access Control
}

\author{
Jason Crampton and Hemanth Khambhammettu \\ Information Security Group, Royal Holloway, University of London
}

\begin{abstract}
User delegation is a mechanism for assigning access rights available to a user to another user. A delegation operation can either be a grant or transfer operation. Delegation for role-based access control models have extensively studied grant delegations. However, transfer delegations for role-based access control have largely been ignored. This is largely because enforcing transfer delegation policies is more complex than grant delegation policies. This paper, primarily, studies transfer delegations for role-based access control models. We also include grant delegations in our model for completeness. We present various mechanisms that authorise delegations in our model. In particular, we show that the use of administrative scope for authorising delegations is more efficient than using relations. We also discuss the enforcement and revocation of delegations. Finally, we compare our work with relevant work in the literature.
\end{abstract}

\section{Introduction}

Role-based access control (RBAC) is being increasingly recognized as an efficient access control mechanism that facilitates security administration 11. Roles are identified with various job functions in an organization and users are assigned to roles based on their job responsibilities and qualifications. Permissions are associated with roles. Users acquire permissions through the roles allocated for them. This feature of role-based models greatly simplifies the management of permissions.

Delegation is a mechanism of assigning access rights to a user. Delegation may occur in two forms: administrative delegation and user delegation. An administrative delegation allows an administrative user to assign access rights to a user and does not, necessarily, require that the administrative user possesses the ability to use the access right. A user delegation allows a user to assign a subset of his available rights to another user. However, a user delegation operation requires that the user performing the delegation must posses the ability to use the access right. Furthermore, like Schaad, we believe that an administrative delegation operation is often long-lived and more durable (permanent) than a user delegation operation that is short-lived (temporary) and intended for a specific purpose [2, Chapter 7, Page 117]. This paper studies user delegation. In the rest of the paper, 'delegation' is always understood to be as 'user delegation' unless stated otherwise. The user who performs a delegation is referred to as a 'delegator' and the user who receives a delegation is referred to as a 'delegatee'.

D. Gollmann, J. Meier, and A. Sabelfeld (Eds.): ESORICS 2006, LNCS 4189, pp. 174-191 2006.

(C) Springer-Verlag Berlin Heidelberg 2006 
Rights can be delegated in two ways in RBAC: by delegating roles or by delegating individual permissions. Delegating a permission $p$ gives the delegatee the ability to use $p$. However, delegating a role $r$ gives the delegatee the ability to act in role $r$. That is, the delegatee is authorized for role $r$ (and thereby gains the ability to use permissions assigned to role $r$ and roles that are junior to $r$ ). In particular, we note that individually delegating all permissions explicitly assigned to a role $r$ does not authorize the delegatee to act in role $r$.

Broadly, delegation of privileges may be classified into (at least) two kinds: grant and transfer [3. A grant delegation model, following a successful delegation operation, allows a delegated access right to be available to both the delegator and delegatee. As such, this is a monotonic model, in which available authorizations are only increased due to successful delegation operations. However, in transfer delegation models, following a successful delegation operation, the ability to use a delegated access right is transferred to the delegatee; in particular, the delegated access right is no longer available to the delegator.

Grant delegation models are, primarily, concerned with allowing the delegatee to use the delegated access right. However, in transfer delegation models, besides allowing the delegatee to use the delegated access right, we must prevent the use of the delegated access right by the delegator. It is this feature that makes it more difficult to enforce transfer delegation policies in most access control frameworks 2,4. Furthermore, it can be easily seen that, in grant delegation models the availability of access rights increases monotonically with delegations. While some business requirements may support grant delegations, it is often desirable that sensitive access rights may not be available to a large number of users (at any given time). Such requirements are usually expressed as cardinality constraints in an access control policy [5, 6. Transfer delegation policies prove to be more useful when an access control policy specifies cardinality limits on the availability of access rights between users.

Consider, for example, an access control policy that requires the co-operation of $k$ users to accomplish a given task. The unavailability of (at least) one of the users, which can be for several reasons, makes it impossible to complete the task. A desirable solution would be for users to be able to delegate the access right to another user, who may act on behalf of the former user. In the above example, when assumed that the access control policy specifies that the right $r$, that is required to complete the task, is available to no more than $k$ users, the reference monitor will always deny an attempt to delegate the right $r$, using grant delegation, to another user to prevent violation of the policy. Such scenarios require that the delegation be made using transfer delegation.

Most works that studied delegation in the context of role-based models are grant delegation models $[3,7,8,9,10,11,12,13$. To our knowledge no work has studied temporary transfer delegation for role-based models. This paper, primarily, aims to study transfer delegation for role-based models. Role hierarchies are an important reason for the wide interest in role-based models. Hence, it is natural to consider role hierarchies when studying any aspect of rolebased models. The semantics of transfer delegations become more complex when 
role-hierarchies are considered. We develop a comprehensive delegation model for role-based systems that provides support for both grant and transfer delegation policies. In this paper, we study delegation in the context of both $\mathrm{RBAC}_{0}$ model (flat roles) and $\mathrm{RBAC}_{1}$ model (hierarchical roles) of the RBAC96 family of models [1].

The rest of the paper is organized as follows. In the next section, we develop the background for the rest of the paper and define semantics for grant and transfer delegations. Section 3 describes the mechanism for authorising delegations. Section 4 presents enforcement of delegations. A comparison of our work with related work in the literature is given in Sect. 5. We conclude this work and discuss future work in Sect. 6.

\section{Role-Based Delegation}

The theoretical development of role-based access control and its standardization has been strongly influenced by the RBAC96 family of models [1]. For this reason, we consider delegation within the context of the RBAC96 family of models. In the next section, we introduce some important prerequisite concepts, including the relevant features of RBAC96 and the concept of administrative scope, which is the building block of the RHA family of administrative models [14.

\subsection{Preliminaries}

RBAC96. $\mathrm{RBAC}_{0}$ is the simplest model and defines a set of roles $R$, a set of permissions $P$, a set of users $U$, a permission-role assignment relation $P A \subseteq$ $P \times R$, and a user-role assignment relation $U A \subseteq U \times R$. We denote the set of roles explicitly assigned to a user $u$ by $R_{u}$; that is, $R_{u}=\{r \in R:(u, r) \in U A\}$ and the set of roles explicitly assigned to a permission $p$ by $R_{p}$; that is, $R_{p}=$ $\{r \in R:(p, r) \in P A\}$.

$\mathrm{RBAC}_{1}$ introduces the concept of a role hierarchy $R H \subseteq R \times R$. The graph of the relation $R H$ is acyclic and the transitive reflexive closure of $R H$ defines a partial order on $R$.

We write $r \leqslant r^{\prime}$ in preference to $\left(r, r^{\prime}\right) \in R H^{*}$ (the transitive reflexive closure of $R H)$. We may also write $r^{\prime} \geqslant r$ whenever $r \leqslant r^{\prime}$. We write $\downarrow r$ to denote the set $\left\{r^{\prime} \in R: r^{\prime} \leqslant r\right\}$ and $\downarrow R^{\prime}$ to denote $\bigcup_{r^{\prime} \in R^{\prime}} \downarrow r^{\prime}$. We write $\uparrow r$ to denote the set $\left\{r^{\prime} \in R: r^{\prime} \geqslant r\right\}$ and $\uparrow R^{\prime}$ to denote $\bigcup_{r^{\prime} \in R^{\prime}} \uparrow r^{\prime}$.

Access control decisions are granted within the context of a user session, which is determined by the set of roles that a user activates. This set of roles is a subset of the roles for which the user is authorized directly by the $U A$ relation and indirectly by the role hierarchy. We denote a session for user $u$ by $S_{u} \subseteq \downarrow R_{u}$. A user $u$ is permitted to invoke a permission $p$ if there exists an activated role $r \in S_{u}$ and a role $r^{\prime} \in R$ such that $\left(p, r^{\prime}\right) \in P A$ and $r^{\prime} \leqslant r$.

Administrative scope. While RBAC96 is widely regarded as the de facto standard for role-based access control, there is less consensus regarding rolebased administration. There are three approaches that are regarded as being 
relatively mature and sophisticated [15]: the ARBAC97 model [16], which is the administrative counterpart of RBAC96; the RHA family of models [14]; and the role control center [15. Since delegation can be regarded as "lightweight userbased administration", it is natural that ideas from role-based administration may be useful in models for delegation. Indeed, the can_delegate relation in RDM2000 is very similar in structure and meaning to the can_assign relation from ARBAC97 [12]. In this section, we introduce the concept of administrative scope from the RHA model and generalize its definition for use in our delegation model.

Definition 1 (Crampton and Loizou [14]). Let $r \in R$ and define $\sigma(r)=$ $\{s \leqslant r: \uparrow s \subseteq \downarrow r \cup \uparrow r\}$. We say that $\sigma(r)$ is the administrative scope of $r$.

In Fig. 1(a), for example, $\sigma(b)=\{d\} ; g \notin \sigma(b)$, because $e>g$ and $e$ is not comparable to $b$. Administrative scope defines a sub-hierarchy forming a natural unit of administration for the role $r$.

The success of any administrative operation in the RHA model is determined by the inclusion (or otherwise) of any role parameters in the requesting role's administrative scope. Hence, a user assigned to role $b$ could add a new role $i$ with parent role $d$, for example, but could not add a new role $k$ with parent role $g$.

Administrative scope has been shown to be a far more flexible approach to role-based administration than ARBAC97 [14]. As such, it is unsurprising that it turns out to have considerable advantages in role-based delegation. However, it will be necessary to compute the administrative scope of a role in different partially ordered sets, each of which is a sub-hierarchy of the role hierarchy. Hence, we extend our notion of administrative scope to include two parameters: a partially ordered set $X$ and an element $x \in X$. We write $\sigma(x, X)$ to denote the administrative scope of $x$ computed in partially ordered set $X$. In practice $X$ will be a subset of $R$. We now discuss the two most important cases.

- If a user $u$ is assigned to a set of roles $R_{u}$, this induces a user view $\downarrow R_{u}$ of the role hierarchy containing all the roles to which $u$ is implicitly assigned (via the user-role and role hierarchy relations). This user view is important in defining which roles a user retains following the delegation of a role. In this case, when $u$ delegates $r$, we compute $\sigma\left(r, \downarrow R_{u}\right)$ (see Proposition 2).

- Similarly, a set of activated roles $S_{u} \subseteq \downarrow R_{u}$ defines a session view $\downarrow S_{u}$, which is useful in defining the roles available to a user following certain types of transfer delegation. In this case, when $u$ delegates $r$, we compute $\sigma\left(r, \downarrow S_{u}\right)$ (see Proposition 3).

\subsection{Delegation Operations}

We now describe the characteristics of delegation operations in RBAC96. The grant operation is well understood and has been described in several earlier papers $[3,7,8,9,10,11,12,13$. We include it here for completeness. We define three different types of transfer operations in the context of $\mathrm{RBAC}_{1}$. 


\section{$\operatorname{RBAC}_{0}$}

$\operatorname{grantR}_{0}(u, v, r)$ : The delegator $u$ grants the role $r$ to delegatee $v$. The delegator may continue to use $r$.

$\operatorname{grantP}_{0}(u, v, p)$ : The delegator $u$ grants the permission $p$ to delegatee $v$. The delegator may continue to use $p$.

$\mathrm{xferR}_{0}(u, v, r)$ : The delegator $u$ transfers the role $r$ to delegatee $v$. The delegator may no longer use $r$.

$\mathrm{xferP}_{0}(u, v, p)$ : The delegator $u$ transfers the permission $p$ to delegatee $v$. The delegator may no longer use $p$.

\section{$\operatorname{RBAC}_{1}$.}

grantR $R_{1}(u, v, r)$ : The delegator $u$ grants the role $r$ to delegatee $v$. The delegator may continue to use $r$. The delegatee acquires the right to use all roles in $\downarrow r$.

$\operatorname{grantP}_{1}(u, v, p)$ : The delegator $u$ grants the permission $p$ to delegatee $v$. The delegator may continue to use $p$.

$\mathrm{xferP}_{1}(u, v, p)$ : The delegator $u$ transfers the permission $p$ to delegatee $v$. The delegator may not continue to use $p$.

xferRstrong $(u, v, r)$ : The delegator $u$ transfers the role $r$ to delegatee $v$. The delegator may not use any role in $\downarrow r$. The delegatee acquires the right to use all roles in $\downarrow r$. We call this strong transfer of a role from the delegator to the delegatee.

xferRstatic $(u, v, r)$ : The delegator $u$ transfers the role $r$ to delegatee $v$. The delegator may not use $x \in \downarrow r$ unless there exists a role $r^{\prime}$ such that $\left(u, r^{\prime}\right) \in$ $U A$ and $x \leqslant r^{\prime}$. (Informally, $u$ retains the use of a role $x$ if there is an alternative path from $x$ to a role to which $u$ is assigned.) We call this static weak transfer of a role from the delegator to the delegatee. As before, the delegatee acquires the right to use all roles in $\downarrow$ r.

xferRdynamic $(u, v, r)$ : The delegator $u$ transfers the role $r$ to delegatee $v$. The roles available to the delegator are determined by the roles he activates in a session. The delegator may not use $x \in \downarrow r$ unless there exists a role $r^{\prime}$ such that $u$ has activated $r^{\prime}$ in the current session and $x \leqslant r^{\prime}$. (Informally, $u$ regains the use of a role $x$ if there is an alternative path from $x$ to a role that $u$ has activated in her session.) We call this dynamic weak transfer of a role from the delegator to the delegatee. As before, the delegatee acquires the right to use all roles in $\downarrow r$.

Given the above definitions, the following results are used as a basis for deciding whether the delegator is allowed to use a role following a successful transfer delegation operation. We discuss the enforcement of the consequences of transfer delegations in Sect. 4.

Proposition 2. If $u$ has performed a static weak delegation of role $r$, then $u$ is denied access to all roles in $\sigma\left(r, \downarrow R_{u}\right)$, where $R_{u}$ denotes the set of roles assigned to $u$. 
Proof. Suppose $u$ cannot use role $x$. Then $x \leqslant r$ and there does not exist a role $r^{\prime}$ such that $\left(u, r^{\prime}\right) \in U A$ and $x \leqslant r^{\prime}$. Hence, $\uparrow x \subseteq \downarrow r \cup \uparrow r$ in $\downarrow R_{u}$. The result follows.

Proposition 3. If $u$ has performed a dynamic weak delegation of role $r$, then $u$ is denied access to all roles in $\sigma\left(r, \downarrow S_{u}\right)$, where $S_{u}$ is the set of roles activated by $u$.

Proof. The proof is analogous to that of Proposition 2.

Proposition 4. Let $R_{\text {strong }}, R_{\text {dynamic }}$ and $R_{\text {static }}$ denote the set of roles denied to a user following the operations $\mathrm{xferRstrong}(u, v, r)$, xferRdynamic $(u, v, r)$ and $\mathrm{xferRstatic}(u, v, r)$, respectively. Then $R_{\text {strong }} \subseteq R_{\text {dynamic }} \subseteq R_{\text {static }}$.

Proof. $R_{\text {strong }}=\downarrow R_{u} \backslash \downarrow r, R_{\text {dynamic }}=\downarrow R_{u} \backslash \sigma\left(r, \downarrow S_{u}\right)$ and $R_{\text {weak }}=\downarrow R_{u} \backslash$ $\sigma\left(r, \downarrow R_{u}\right)$. Now $\sigma(r) \subseteq \downarrow r$ irrespective of the sub-hierarchy in which $\sigma$ is computed. Hence $R_{\text {strong }} \subseteq R_{\text {dynamic }}$. Moreover, $S_{u} \subseteq R_{u}$ and it is easy to see that this implies that $\sigma\left(r, \downarrow R_{u}\right) \subseteq \sigma\left(r, S_{u}\right)$ (since if $r^{\prime} \geqslant x$ for some $x \in \downarrow r$ and some $r^{\prime} \in S_{u}$, then $r^{\prime} \in R_{u}$ ).

Consider the role hierarchy depicted in Fig. 1 We assume that some user $u$ is assigned to roles $b$ and $f$. In the diagram, unfilled nodes represent roles that are available to $u$ (and filled nodes represent nodes that are not available). Each diagram represents different views of the role hierarchy: Fig. 1(a) illustrates the whole hierarchy; Fig. 1(b) illustrates $u$ 's view of the hierarchy; and Fig.s 1(d) and 1(e) illustrate two different session views, one in which only role $b$ is activated and one in which only role $f$ is activated.

The user delegates role $d$, represented by the square node, to another user. Figure 1(b) illustrates the roles that are denied to the user in the event that strong transfer is used to delegate the role. If we are using dynamic weak transfer, then the ability to use role $h$ is determined by whether the user activates role $f$, as shown in Fig. 1(d) and Fig. 1(e) If we are using static weak delegation, the role $h$ is always available, as depicted in Fig. 1(c).

\section{Controlling Delegation}

We assume that an access control policy specifies whether delegation of a role or permission is permitted. We also assume the presence of a reference monitor that evaluates such access control policies to determine whether a delegation operation is permitted. There are two issues involved in the specification of delegations:

- Is a user (delegator) authorized to delegate a role or permission that is available to him?

- Can a role or permission be delegated to a user (delegatee)?

In this section, we describe two new mechanisms for authorizing delegations, and discuss their advantages over existing approaches. 


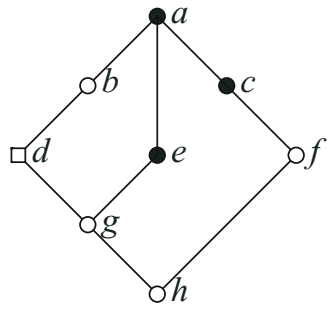

(a) $R$

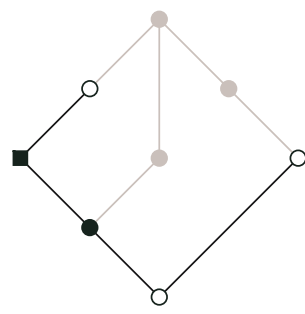

(c) Weak static transfer

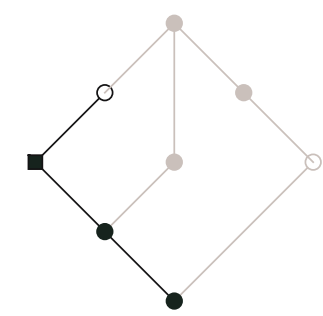

(d) Weak dynamic transfer: $b$ activated

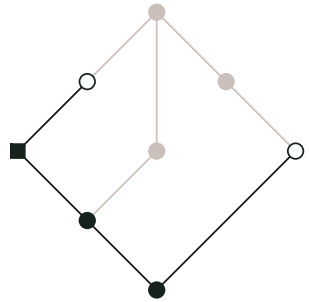

(b) Strong transfer

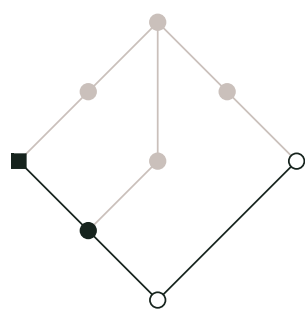

(e) Weak dynamic transfer: $f$ activated

Fig. 1. Transfer delegation patterns for role $d$

\subsection{Delegation Relations}

Role delegation relation. We introduce two relations that authorize role delegations in a role-based system: can-delegate and can-receive. The binary relation can-delegate specifies the set of roles that can be delegated by a delegator and the relation can-receive authorizes delegation of a role to a delegatee.

The relation can-delegate $\subseteq R \times R$ specifies whether a user is authorized to delegate a role. $\left(r, r^{\prime}\right) \in$ can-delegate specifies that a user $u$ is authorized to delegate role $r^{\prime}$, if $r \in R_{s}$, where $R_{s}$ denotes the set of roles that are active in $u$ 's current session $s$. For example, in Fig. 1(a), if $(b, d) \in$ can-delegate then $u$ may delegate role $d$ if $b \in R_{s}$.

We define a constraint on the tuples in the can-delegate relation that guarantees that no user can delegate a role $r$ that the user is not assigned to. Specifically, we require that if $(r, s) \in$ can-delegate then $r \geqslant s$. In our example, in Fig. 1(a) an attempt to add the tuple $(b, d)$ to the can-delegate relation will succeed since $b \geqslant d$. However, an attempt to add the tuple $(d, c)$ to the can-delegate relation will fail since $d \ngtr c$. 
A delegatee must satisfy a set of conditions, usually role memberships, in order to be authorized to receive a delegation. A delegation condition specifies the conditions that must be satisfied by the delegatee to receive a delegation. We adopt the notation of a SARBAC constraint to model a delegation condition [14]. Let $R^{\prime}=\left\{r_{1}, \ldots, r_{k}\right\}$ be a subset of $R$ and let $\bigwedge R^{\prime}$ denote $r_{1} \wedge \cdots \wedge r_{k}$. We model a delegation condition as $\bigwedge C$ for some $C \subseteq R$. A delegation condition $\bigwedge C$ is said to be satisfied by a user $v$ if $C \subseteq \downarrow R_{v}$. In other words, the condition $\wedge C$ is satisfied by the delegatee if he is assigned to all the roles in $C$. The relation can-receive $\subseteq R \times C$ specifies whether a user is authorized to receive a role delegation. $(r, C) \in$ can-receive means a delegatee $v$ may receive a delegation of role $r$ provided that $v$ satisfies $\bigwedge C$.

It may be more efficient to limit the set of roles that a delegatee may receive due to a successful delegation. For example, in Fig. 1(a) is it reasonable for a delegation request to succeed that delegates a role $c$ to a delegatee $v$ who currently is assigned to role $g$ ? We may often require that such delegations are not allowed, since $v$ may lack sufficient expertise to efficiently perform the job requirements of role $c$. Essentially, we require that a delegation always results in a natural progression for the delegatee with respect to the role hierarchy. We formalize the above requirement by defining the following constraint on the tuples in the can-receive relation: $(r, C) \in$ can-receive then for all $r^{\prime} \in C$ we require that $r^{\prime} \leqslant r$. Note that this constraint does not apply if $r$ has no junior roles (that is, $r$ is a leaf node in the hierarchy). In our example, $(c,\{g\})$ is not a permissible entry in can-receive; $(c,\{f\})$, in contrast, is a permissible entry.

In summary, the role-based reference monitor refers to the can-delegate and can-receive relations to establish whether a delegation operation can succeed. A request by $u$ to delegate $r$ to $v$ will succeed only if there exists $\left(r^{\prime}, r\right) \in$ can-delegate such that $r^{\prime} \in R_{s}$, where $s$ is $u$ 's current session and a tuple $(r, C) \in$ can-receive such that $v$ satisfies $\wedge C$. In our example, let us assume that $(b, d) \in$ can-delegate, $(d,\{g\}) \in$ can-receive, $(u, b) \in U A$ and $(v, g) \in$ $U A$. Then an attempt by $u$ to delegate role $d$ to $v$ will succeed.

Permission delegation relation. We define a relation can-delegatep $\subseteq R \times$ $P$ that specifies the set of permissions that can be delegated by a user $u .(r, p) \in$ can-delegatep specifies that a delegator $u$ may delegate a permission $p$ provided that there exists $r \in R_{s}$. Similar to the can-delegate relation, we define a constraint on the can-delegatep relation that guarantees that no user $u$ can delegate a permission $p$ that is not available to $u$. In other words, we require that if $(r, p) \in$ can-delegatep then there exists a role $r^{\prime}$ such that $\left(p, r^{\prime}\right) \in P A$ and $r^{\prime} \leqslant r$.

The relation can-receivep $\subseteq P \times C$ specifies whether a user is authorized to receive a permission delegation, where $C$ is a delegation condition as defined above. $(p, C) \in$ can-receivep means that a delegatee $v$ is authorized to receive a delegation of a permission $p$ if $v$ satisfies $\bigwedge C$. Similar to the can-receive relation, we define a constraint on the can-receivep relation that ensures that a delegation always results in a natural progression for the delegatee with respect 
to the role hierarchy. If $(p, C) \in$ can-receivep then there must exist $r^{\prime} \in C$ and $r \in R$ such that $(p, r) \in P A$ and $r^{\prime} \leqslant r$.

\subsection{Using Administrative Scope}

The use of relations for controlling delegations, discussed above, has been used extensively in the literature mainly perhaps because of its simplicity [7, 12, 13. The use of relations is simple, if we assume that RBAC relations, such as the role hierarchy relation $R H$, remain static. However, updates to the RBAC relations may lead to inconsistencies in the can-delegate and can-receive relations. Such inconsistencies are explained in detail in Sect. 3.3. For now, we note that the dynamic nature of various RBAC components increases the complexity of managing the relations that are used for controlling delegations. It is our belief that the mechanism used for controlling delegations must be simple and able to implicitly handle any updates to RBAC relations. In this section, we suggest an alternative method for controlling delegations, which dynamically handles updates to RBAC relations using the concept of administrative scope [14].

We limit the extent to which a user can delegate roles and permissions using the administrative scope of the roles(s) activated by the user. Specifically, we define the administrative scope of a session $s$ to be

$$
\sigma(s)=\bigcup_{r \in S} \sigma(r) .
$$

Then in order for the delegation of role $r$ by user $u$ to succeed we require that $r \in \sigma(s)$, where $s$ is $u$ 's current session. In other words, $u$ can only delegate roles that are within his administrative scope. Similarly, in order for the delegation of permission $p$ by user $u$ to succeed we require that there exists $r \in s$ such that $(p, r) \in P A$ and $r \in \sigma(s)$.

We now consider what criteria the delegatee must satisfy to be able to receive a delegation. Informally, for the delegation of $r$ to $v$ to succeed, we require that $v$ is already "sufficiently authorized". Now, the delegation of role $r$ means that the delegatee is authorized for all roles $r^{\prime} \leqslant r$. These observations lead to the idea that the delegatee should already be assigned to any roles outside the delegator's administrative scope that the delegatee will acquire as a result of the delegation. More formally, for the delegation of role $r$ to user $v$ by user $u$ to succeed we require that for all $r^{\prime}<r$ such that $r^{\prime} \notin \sigma(s)$, there exists $r^{\prime \prime}$ such that $r^{\prime} \leqslant r^{\prime \prime}$ and $\left(v, r^{\prime \prime}\right) \in U A$.

In our example, Fig. 1(a), $u$ (who is assigned to $b$ and $f$ ) may delegate role $d$ since $d \in \sigma(b)$. Moreover, the delegation of $d$ to user $v$ will only succeed if $v$ is already assigned to role $g$. Note, however, that a user $w$ who is assigned (only) to role $f$ will not be able to receive the delegated role $d$ (from $u$ ) because $g \notin \sigma(s)$, $g<d$ and $w$ is not assigned to $g$.

Administrative scope implicitly deals with any updates to various RBAC relations, in particular the role hierarchy relation $R H$, since administrative scope of a session $s$ is computed, dynamically, with respect to the role hierarchy. Consider, for example, Fig. 2(a) and Fig. 2(b) that depicts our original role hierarchy 
and a role hierarchy that is obtained after deleting an edge between roles $d$ and $b$. In the original hierarchy, if a user $u$ has activated $b$ in a session $s$ then $u$ is authorized to delegate role $d$ since $d \in \sigma(s)$. However, following the edge deletion operation, $u$ is no longer able to delegate $d$ since $d \notin \sigma(s)$. In other words, the success of delegation operations adapts in a natural and transparent way to changes in the structure of the role hierarchy. Figure 2(c) depicts the role hierarchy obtained after deleting an edge between roles $g$ and $d$. In the original hierarchy, the delegatee $v$, who is assigned to role $g$, can receive a delegation of role $d$ since $g<d$. Following the edge deletion operation, $v$ will not be able to receive a delegation of $d$ because $g \nless d$. Note that in Fig. 2(c) there are no roles less than $d$. This observation leads to the result that no user can receive a delegation of a role $\{r \in R: \downarrow r \subseteq \uparrow r \cap \downarrow r\}$.

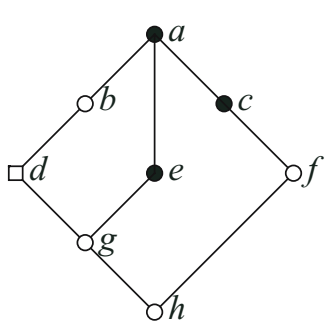

(a) $R$

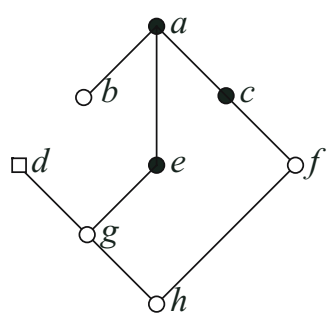

(b)

After

deleteEdge $(d, b)$

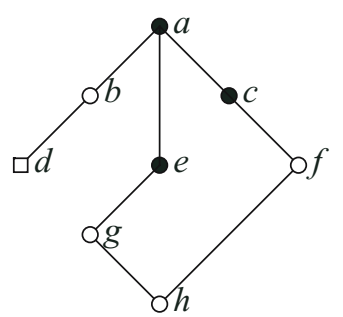

(c) deleteEdge $(g, d)$

Fig. 2. Role hierarchies before and after edge deletion

\subsection{Discussion}

Most work in the literature uses a single relation to control delegation [7, 12, 13 . This relation encodes conditions on both the delegator and delegatee. We have proposed an alternative approach where the above issues are dealt with independently. There are several advantages in dealing with these issues separately. Primarily, it eases the management of delegation specification. Furthermore, such an approach employs separation of tasks, thus, making the model less error prone while updating delegation policies. For example, if we wish to revoke the authority of a role $r$ to perform any delegations, then appropriate tuples are deleted from the can-delegate relation. Note that such an operation does not require any updates to the can-receive relation that authorizes a delegation to the delegatee. Similarly, if we require that a permission $p$ may no longer be delegated to a delegatee who satisfies a condition $\bigwedge C$, we only delete necessary tuples from the can-receivep relation.

The use of relations to control delegation is common to most existing approaches, mainly perhaps because of its simplicity [7, 12, 13. The use of relations 
is appropriate, if we assume that RBAC structures such as the role hierarchy remain static. However, updates to the role hierarchy may lead to inconsistencies in the can-delegate and can-receive relations. Consider, for example, Fig. 2(a) and Fig. 2(b) that depict an original role hierarchy and a role hierarchy obtained after deleting an edge between roles $d$ and $b$. If $(b, d) \in$ can-delegate it will need to be explicitly deleted following the deletion of this edge. Similar considerations apply to the operations that involve revocation of a permission $p$ assigned to $b$. Similarly, if $(b,\{d\}) \in$ can-receive, then we must delete this entry following the deletion of the edge between $b$ and $d$. In summary, the can-delegate and can-receive relations must be updated after updates to certain RBAC relations to prevent inconsistencies.

Hence the advantages of using relations for controlling delegations are limited if we allow updates to RBAC relations. In contrast, administrative scope is a dynamic model and implicitly deals with any updates to RBAC relations. It is important to note that, following successful updates to RBAC relations, no explicit updates are required with the use of administrative scope to resolve any inconsistencies involved in controlling delegations. This is because administrative scope (and hence delegation) is determined by the structure of the role hierarchy. In short, the use of administrative scope greatly simplifies delegation in the presence of dynamic RBAC structures.

Furthermore, the administrative scope model for controlling delegations preserves the separation of conditions on delegator and delegatee that we introduced in our relation-based model. The issues that are involved in controlling delegations, such as specifying the roles that a user is authorized to delegate and receive, are still dealt with independently. Note also that the constraint we use to limit the increase of power of the delegatee can be framed very elegantly using administrative scope and existing RBAC relations.

\section{Enforcing Delegation Constraints}

Enforcing delegation operations that grant a role or permission to a user is quite straightforward as it is a monotonic action. That is, the set of roles or permissions for each user either stays the same or increases. Delegation operations that transfer a role or permission are rather more difficult. In this section, we introduce three relations that are used to guarantee that delegation operations are enforced correctly. The model described in this section can be used for both grant and transfer delegations. To our knowledge, this is the first treatment of the consequences of temporary transfer delegation policies for access control in role-based systems. This is mainly due to the fact that there is much to say on the subject when grant delegations, but not transfer delegations, are allowed in role-based models.

\subsection{The Delegation History Relation}

The delegation history $(D H)$ relation is used to record all delegations that have been made. The $D H$ relation is used by the delegators and administrative users 
for administrative purposes. Typically, such activities include auditing and revoking delegations.

A $D H$ tuple has the form $(i, u, v, o, C, M)$ where $i$ is an identifier, $u$ is a delegator, $v$ is the delegatee, $o$ is an object or target of the delegation that can either be a set of roles $R^{\prime} \subset R$ and/or a set of permissions $P^{\prime} \subseteq P, C$ is the set of conditions, from the can-receive relation, that must be satisfied by the delegatee and $M$ is a delegation mask. A delegation mask records various internal details of a delegation.

A delegation mask $M$ contains five bits which are used to record certain details of the delegation. More specifically, a delegation mask has the form $b_{4} b_{3} b_{2} b_{1} b_{0}$, where $b_{i} \in\{0,1\}, b_{4}$ specifies whether the delegated object can be further delegated $1, b_{3}$ specifies whether the delegated object is a role or permission, $b_{2}$ specifies whether the delegation is static or dynamic, $b_{1}$ specifies whether the delegation is strong or weak, and $b_{0}$ specifies whether the delegation is grant or transfer. The characteristics of a delegation mask are summarized in Table 1. and some examples of masks are shown in Table 2 together with the commands that would give rise to such a mask.

Note that we require the delegator to explicitly set a few values of the bits in the delegation mask while performing a delegation operation. In particular, we require that the bits $b_{0}, b_{1}$ and $b_{2}$ are set by the delegator, while the bits $b_{3}$ and $b_{4}$ are deduced by the access control enforcement system.

Table 1. Delegation mask bit values

\begin{tabular}{|l|l|l|l|l|l|}
\hline & $b_{4}$ & $b_{3}$ & $b_{2}$ & $b_{1}$ & $b_{0}$ \\
\hline 0 & undelegatable & role & static & strong & grant \\
1 & delegatable & permission & dynamic & weak & transfer \\
\hline
\end{tabular}

\subsection{Temporary Delegation Relations}

We also introduce two relations tempU A and tempPA, which record temporary user-role and user-permission assignments that arise from delegation operations. tempUA contains tuples of the form $(i, u, r, s)$, where $s \in\{-,+\}$ and $i$ identifies a tuple in the $D H$ relation. The meaning of the tuple $(i, v, r,+)$ is analogous to $(v, r) \in U A$; such a tuple would arise as a result of a grant or transfer of role $r$ to the delegatee $v$. In contrast, a tuple of the form $(i, u, r,-)$ means that $u$ is prohibited from activating role $r$; such a tuple only arises when $u$ transfers $r$ to $v$. The precise set of roles that are unavailable for the delegator $u$ depends on the transfer type used for delegation (strong, weak static or weak dynamic).

tempPA contains tuples of the form $(i, u, p, s)$, where $s \in\{-,+\}$ and $i$ identifies a tuple in the $D H$ relation. The tuple $(i, v, p,+)$ means that $v$ is allowed

\footnotetext{
${ }^{1}$ An extra boolean-valued parameter can be added to each of the delegation commands; this parameter would be used to set this bit.
} 
Table 2. Examples of valid delegation mask values, their semantics, and associated commands

\begin{tabular}{|l|l|l|}
\hline Mask & Delegation semantics & Command \\
\hline 00xx0 & undelegatable, role, grant & grantR \\
10x01 & delegatable, role, strong, transfer & xferRstrong \\
00011 & undelegatable, role, static, weak, transfer & xferRstatic \\
10111 & delegatable, role, dynamic, weak, transfer & xferRdynamic \\
$11 \times x 0$ & delegatable, permission, grant & grantP \\
$11 \times 01$ & delegatable, permission, strong, transfer & xferPstrong \\
01111 & undelegatable, permission, dynamic, weak, transfer & xferPdynamic \\
11011 & delegatable, permission, static, weak, transfer & xferPstatic \\
\hline
\end{tabular}

to use $p$; such a tuple would arise as a result of a grant or transfer of permission $p$ to the delegatee $v$. A tuple of the form $(i, u, p,-)$ means that $u$ is prohibited from invoking permission $p$; such a tuple only arises when $u$ transfers $p$ to $v$.

For simplicity, we write $\left(i, u, R^{\prime}, s\right)$ to denote $\left\{\left(i, u, r^{\prime}, s\right): r^{\prime} \in R^{\prime}\right\}$. We also write $\left(i, u, P^{\prime}, s\right)$ to denote $\left\{\left(i, u, p^{\prime}, s\right): p^{\prime} \in P^{\prime}\right\}$. We now describe the effects on various relations following the successful execution of a delegation operation. We focus our attention on the more difficult case of $\mathrm{RBAC}_{1}$.

Successful delegation operations are recorded in the delegation history relation $D H$. Hence, following any delegation of $o$ by $u$ to $v$, we have $D H \leftarrow D H \cup$ $\{(i, u, v, o, C, M)\}$. In addition we have the following operational semantics:

$\operatorname{grantR}_{1}(u, v, r): u$ grants role $r$ to $v$. Such a delegation requires that the delegatee $v$ is allowed to use role $r$. Hence, tempU $A \leftarrow \operatorname{temp} U A \cup\{(i, v, r,+)\}$.

$\operatorname{grantP}_{1}(u, v, p): u$ grants permission $p$ to the delegatee $v$. Then, permission $p$ must be available to the delegatee $v$. Hence, tempPA $\leftarrow \operatorname{tempPA\cup }$ $\{(i, v, p,+)\}$.

$\operatorname{xferP}_{1}(u, v, p)$ : delegator $u$ transfers the authority to use permission $p$ to the

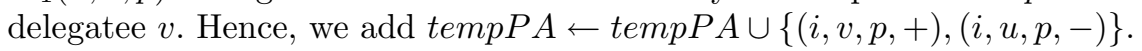

xferRstrong $(u, v, r): u$ performs a strong transfer of role $r$ to $v$. The delegator $u$ may not use any role in $\downarrow r$ and the delegatee $v$ acquires the right to use all roles in $\downarrow r$. Hence, temp $U A \leftarrow \operatorname{temp} U A \cup\left\{(i, v, r,+),\left(i, u, R^{\prime},-\right)\right\}$, where $R^{\prime}=\downarrow r$.

xferRstatic $(u, v, r): u$ performs a static weak transfer of role $r$ to $v$. The delegator $u$ may not use a role $x \in \downarrow r$ unless there exists a role $r^{\prime}$ such that $\left(u, r^{\prime}\right) \in U A$ and $x \leqslant r^{\prime}$. Hence, by Proposition [2, tempUA $\leftarrow$ tempU $A \cup$ $\left\{(i, v, r,+),\left(i, u, R^{\prime},-\right)\right\}$, where $R^{\prime}=\sigma\left(r, \downarrow R_{u}\right)$.

xferRdynamic $(u, v, r): u$ performs a dynamic weak transfer of role $r$ to $v$. The set of roles available to the delegator $u$ are computed by the roles that 
$u$ activates in a session. Hence, by Proposition 3, tempU $A \leftarrow$ tempUA $\cup$ $\left\{(i, v, r,+),\left(i, u, R^{\prime},-\right)\right\}$, where $R^{\prime}=\sigma\left(r, \downarrow S_{u}\right)$ ?

\subsection{Access Control Decisions}

The role-based reference monitor uses the tempUA and tempPA relations, in addition to the $U A$ and $P A$ relations, to make access control decisions. For example, an attempt by a user $u$ to activate a role $r$ is always denied if there exists a tuple $\left(i, u, R^{\prime},-\right) \in$ temp $U A$ such that $r \in R^{\prime}$; and granted if there exists a tuple $\left(i, u, r^{\prime},+\right) \in \operatorname{temp} U A$ or $\left(u, r^{\prime}\right) \in U A$ such that $r \leqslant r^{\prime}$. Similarly, an attempt to invoke a permission $p$ by $u$ is always denied if $(i, u, p,-) \in \operatorname{tempP} A$; and granted if $(i, u, p,+) \in \operatorname{tempPA}$ or there exist $r, r^{\prime} \in R$ such that $(p, r) \in$ $P A,\left(u, r^{\prime}\right) \in U A$ and $r \leqslant r^{\prime}$.

\subsection{Revocation}

Successful delegations have a specified lifetime or may be revoked before the delegation ends 3 In either case, the tempUA and tempPA relations must be updated to prevent any subsequent use of the delegated access right by the delegatee and, if necessary, to allow the delegator to use the delegated access right. Essentially, we require that when a delegation $d \in D H$ ends or is revoked the tuples $\left(i, u, R^{\prime}, s\right) \in$ tempUA and $\left(i, u, P^{\prime}, s\right) \in$ tempPA are deleted, where $i$ identifies the obsolete delegation $d$. We now describe the effects on various relations when a delegation ends or is revoked.

grant $\mathrm{R}_{1}(u, v, r)$ : When such a delegation ends, the delegatee $v$ is no longer allowed to use role $r$. Hence, tempU $A \leftarrow$ temp $U A \backslash\{(i, v, r,+)\}$.

$\operatorname{grantP}_{1}(u, v, p)$ : permission $p$ must no longer be available for the delegatee $v$. Hence, tempP $A \leftarrow$ tempP $A \backslash\{(i, v, p,+)\}$.

$\operatorname{xfer}_{1}(u, v, p)$ : delegatee $v$ must be prevented from using permission $p$ and the delegator $u$ regains the ability to use the delegated permission $p$. Hence, tempPA $A \leftarrow$ tempPA $A \backslash\{(i, v, p,+),(i, u, p,-)\}$.

xferRstrong $(u, v, r): u$ performs a strong transfer of role $r$ to $v$. The delegator $u$ gets back the right to use any role in $\downarrow r$ and the delegatee $v$ can no longer use any role in $\downarrow r$. Hence, tempUA $\leftarrow$ temp $U A \backslash\left\{(i, v, r,+),\left(i, u, R^{\prime},-\right)\right\}$ where $R^{\prime}=\downarrow r$.

${ }^{2}$ It is important to note that, following a dynamic weak transfer, the roles that are to be prevented for the delegator $u$ are determined by the roles that are active in $u$ 's current session. Hence, it is necessary to add appropriate tuples to the tempUA relation whenever a session is initiated by a user who has performed a weak dynamic delegation.

${ }^{3}$ In a practical implementation, the $D H$ relation would include some information that would determine the lifetime of the delegation: this information might be a start and an end time, or a start time and a duration, for example. 
xferRstatic $(u, v, r): u$ performs a static weak transfer of role $r$ to $v$. Similar to the above delegation, the delegator $u$ gets back the right to use any role in $\downarrow r$ and the delegatee $v$ can no longer use any role in $\downarrow r$. Hence, temp $U A \leftarrow$ tempU $A \backslash\left\{(i, v, r,+),\left(i, u, R^{\prime},-\right)\right\}$ where $R^{\prime}=\sigma\left(r, \downarrow R_{u}\right)$.

xferRdynamic $(u, v, r): u$ performs a dynamic weak transfer of role $r$ to $v$. Again, the delegator $u$ regains the right to use any role in $\downarrow r$ and the delegatee $v$ can no longer use any role in $\downarrow r$. Hence, tempU $A \leftarrow$ temp $U A \backslash$ $\left\{(i, v, r,+),\left(i, u, R^{\prime},-\right)\right\}$ where $R^{\prime}=\sigma\left(r, \downarrow S_{u}\right) 4$

\section{Related Work}

Several delegation models have been proposed for role-based access control [3, 7, 8, 9, 10, 11, 12, 13. The early work of Barka and Sandhu was instrumental in identifying the important considerations for delegation in RBAC [3]. This included the concepts of monotonic and non-monotonic delegation, which correspond to our grant and transfer models. To our knowledge, the work that we present in this paper represents the first attempt to deal properly with the consequences of temporary non-monotonic delegation in RBA 5

Of the work in the literature, the RBDM0, RDM2000 and PBDM models are closest to our work $[7,8,12,13$. RBDM0 is a model for delegating roles, and is based on the $\mathrm{RBAC}_{0}$ model of the RBAC96 family of models [7. Another role delegation model for role-based access control is presented in [8]. RDM2000 defines a rule-based framework for delegation and revocation [12. The model considers role hierarchies and also provides support for multi-step delegations. The PBDM model proposes a delegation model for permissions that supports multi-step delegations 13 .

The RDM2000 model uses a relation can_delegate $\subseteq R \times 2^{R} \times \mathbb{N}$ to authorize delegations, $\mathbb{N}$ is the set of natural numbers. If $(r, C, n) \in$ can_delegate, a delegator acting in role $r$ may delegate any role $r^{\prime} \leqslant r$ to a delegatee $v$ provided $v$ satisfies some role assignment conditions specified by $C ; n$ is used to define the maximum depth of a delegation. A major limitation of this relation is that no constraints are defined on the tuples in the can_delegate relation. That is, $r$ and $C$ are not required to have any relationship with each other. What this means is that the delegatee's power can be arbitrarily increased by a successful delegation. In practice, a delegatee can be assigned to roles for which they lack any relevant expertise or experience.

The PBDM model uses a similarly named relation can_delegate $\subseteq R \times R \times$ $P \times \mathbb{N}$. If $\left(r, r^{\prime}, P^{\prime}, n\right) \in$ can_delegate then a delegator who is assigned to role $r$

\footnotetext{
${ }^{4}$ Recall that, following a dynamic weak transfer of a role $r$, we determine the roles that are not available for the delegator $u$, in a session $s$, by computing $\sigma\left(r, \downarrow S_{u}\right)$ (see Proposition 3). Hence, it may also be necessary to delete appropriate tuples from the tempUA and tempPA relations when the delegator $u$ deactivates a role.

${ }^{5}$ Barka distinguishes between temporary and permanent delegation for role-based delegation models 17. We believe the latter is more correctly viewed as an administrative activity. Barka does not consider temporary non-monotonic delegation policies (which we call transfer delegation policies).
} 
can delegate the set of permissions $P^{\prime}$ to a user who is assigned to a role $r^{\prime}$ with a maximum delegation depth of $n$. Like the RDM2000 model, the can_delegate relation does not require that there is any relationship between $P^{\prime}$ and $r$, which means that it is possible for a delegator $u$ to delegate a permission $p$ that is not available to $u$. As we have already noted, these relation-based approaches to delegation are unlikely to be suitable in environments where the role hierarchy may change.

Unlike the above discussed models, which use a single relation for controlling delegations, we use two different relations: can-delegate and can-receive. Advantages of using different relations for controlling delegations include flexibility, greater control while specifying delegations, ease of management and is less error prone. Furthermore, our model for controlling delegations defines constraints on the can-delegate and can-receive relations. Such constraints ensure that the tuples that are added to the can-delegate and can-receive relations does not give the authority for a delegator $u$ to delegate a right that is not available to $u$ and the rights of a delegatee $v$ can only be incrementally increased and are limited by $v$ 's existing rights.

Hence, we believe our delegation specification model is more conservative (and thus safer), more fine-grained and more manageable than these models [7, 8, 12, 13. However, in Sect. 3.3, we observed that the use of relations for controlling delegations may not be efficient for implicitly handling updates to various RBAC relations. Our model includes an alternative way of controlling delegations using the concept of administrative scope [14. The administrative scope model is dynamic and implicitly handles any updates to RBAC relations, in particular the role hierarchy relation $R H$. We have also described the enforcement of both grant and transfer delegations and dealt with revocations.

A delegation model with restricted permission inheritance is proposed in 9 . The model is based on the idea of dividing a role hierarchy into inter-related role hierarchies. A cascaded role delegation model in the context of decentralized trust management systems is presented in [10. Another model that supports delegation of both roles and permissions is discussed in [11]. We believe that our

Table 3. Delegation characteristics in various delegation models

\begin{tabular}{|l|c|c|c|c|}
\hline Characteristic & RBDM0 & RDM2000 & PBDM & Our model \\
\hline Role delegation & $\checkmark$ & $\checkmark$ & $\checkmark$ & $\checkmark$ \\
Permission delegation & $x$ & $x$ & $\checkmark$ & $\checkmark$ \\
Grant delegation & $\checkmark$ & $\checkmark$ & $\checkmark$ & $\checkmark$ \\
Transfer delegation & $x$ & $x$ & $x$ & $\checkmark$ \\
Controlling delegations & $\checkmark$ & $\checkmark$ & $\checkmark$ & $\checkmark$ \\
Implicit updates & $x$ & $x$ & $x$ & $\checkmark$ \\
Delegation history & $x$ & $\checkmark$ & $x$ & $\checkmark$ \\
Temporary delegation assignments & $\checkmark$ & $\checkmark$ & $\checkmark$ & $\checkmark$ \\
Revocation & $\checkmark$ & $\checkmark$ & $\checkmark$ & $\checkmark$ \\
\hline
\end{tabular}


model can easily be extended to incorporate these concepts. However, none of the above work considers transfer delegation for role-based systems. Table 3 compares the support provided for key delegation features by well known delegation models.

\section{Conclusions and Future Work}

We have developed a comprehensive delegation model for role-based access control that provides support for both grant and transfer delegation policies. This is the first attempt in the literature that extensively studies transfer delegations for role-based access control.

We have discussed two mechanisms for controlling delegations: the relations approach and the administrative scope approach. In particular, we have shown that the concept of administrative scope can be used for authorising delegations and is more effective than relations for delegation in dynamic environments.

We have also presented an enforcement mechanism that supports both grant and transfer delegation policies. Our enforcement model uses a delegation history relation $D H$ and temporary delegation relations, tempUA and tempPA, to guarantee that delegations are enforced correctly. We also discussed various effects on the above relations following a successful delegation operation and corresponding revocations.

An immediate priority in future work is to enrich the revocation mechanism that has been described in this paper. Most importantly, we wish to provide support for cascaded revocations. That is, if a delegation is revoked and this delegation had been used to generate other delegations, then the latter delegations must also be revoked. We may also consider revocation of a delegation when a user is revoked from a role $r \in C$, where $C$ is a delegation condition. Another future work is to consider supporting revocations such as those discussed in [18. Furthermore, we wish to define necessary commands that provide an option to set the delegatable flag in delegation mask. A long term goal is to develop abstract Java classes that implement our delegation model.

\section{References}

1. Sandhu, R., Coyne, E., Feinstein, H., Youman, C.: Role-based access control models. IEEE Computer 29(2) (1996) 38-47

2. Schaad, A.: A Framework for Organisational Control Principles. PhD thesis, The University of York, York, England (2003)

3. Barka, E., Sandhu, R.: Framework for role-based delegation models. In: Proceedings of Twenty Third National Information Systems Security Conference (NISSC'00). (2000) 101-114

4. Aura, T.: Distributed access-rights management with delegation certificates. In: Secure Internet Programming - Security Issues for Distributed and Mobile Objects. Volume 1603 of LNCS. Springer (1999) 211-235

5. Gligor, V., Gavrila, S., Ferraiolo, D.: On the formal definition of separation-of-duty policies and their composition. In: Proceedings of IEEE Symposium on Research in Security and Privacy. (1998) 172-183 
6. Simon, R., Zurko, M.: Separation of duty in role-based environments. In: Proceedings of Tenth IEEE Computer Security Foundations Workshop. (1997) 183-194

7. Barka, E., Sandhu, R.: A role-based delegation model and some extensions. In: Proceedings of Sixteenth Annual Computer Security Applications Conference (ACSAC'00). (2000) 168-177

8. Na, S., Cheon, S.: Role delegation in role-based access control. In: Proceedings of Fifth ACM Workshop on Role-Based Access Control (RBAC'00). (2000) 39-44

9. Park, J., Lee, Y., Lee, H., Noh, B.: A role-based delegation model using role hierarchy supporting restricted permission inheritance. In: Proceedings of the 2003 International Conference on Security and Management (SAM'03). (2003) 294-302

10. Tamassia, R., Yao, D., Winsborough, W.: Role-based casdaded delegation. In: Proceedings of Ninth ACM Symposium on Access Control Models and Technologies (SACMAT'04). (2004) 146-155

11. Wainer, J., Kumar, A.: A fine-grained, controllable, user-to-user delegation method in RBAC. In: Proceedings of Tenth ACM Symposium on Access Control Models and Technologies (SACMAT'05). (2005) 59-66

12. Zhang, L., Ahn, G.J., Chu, B.T.: A rule-based framework for role-based delegation and revocation. ACM Transactions on Information and System Security (TISSEC) 6(3) (2003) 404-441

13. Zhang, X., Oh, S., Sandhu, R.: PBDM: A flexible delegation model in RBAC. In: Proceedings of Eighth ACM Symposium on Access Control Models and Technologies (SACMAT'03). (2003) 149-157

14. Crampton, J., Loizou, G.: Administrative scope: A foundation for role-based administrative models. ACM Transactions on Information and System Security (TISSEC) 6(2) (2003) 201-231

15. Ferraiolo, D., Kuhn, D., Chandramouli, S.: Role-Based Access Control. Artech House, Boston, Massachussetts (2003)

16. Sandhu, R., Bhamidipati, V., Munawer, Q.: The ARBAC97 model for role-based administration of roles. ACM Transactions on Information and System Security 1(2) (1999) 105-135

17. Barka, E.: Framework for Role-Based Delegation Models. PhD thesis, George Mason University, Virginia, USA (2002)

18. Hagström, Å., Jajodia, S., Parisi-Presicce, F.: Revocations- a classification. In: Proceedings of the Fourteenth IEEE Workshop on Computer Security Foundations (CSFW'01). (2001) 44-58 